\title{
Editorial comments: HealthSAGesondheid 2014
}

When taking a closer look at all the articles published in the 2014 edition of HSAG, one can discern the following broad themes: Health professionals and health workers, health challenges people experience, solutions to address health challenges, issues in women's health, and HIV/AIDS. These thus appeared to be the priorities in health research in 2014.

\section{Research on health professionals and health workers}

Eight papers examined this theme. One paper examined nurses' knowledge of chronic disease management. A survey on their knowledge was used as an intervention. After the intervention it was found that nurses demonstrated a significant improvement in their knowledge of chronic diseases. Another paper examined the compliance of occupational nurses' practice. The results of this research study showed that more attention should be paid to compliance. The topic of in-service needs for psychiatric nurses was explored in another paper where it was found that psychiatric nurses need ongoing in-service training to enable them to provide quality patient care. Two articles focused on doctors. One article looked at the experiences of British general medical practitioners participating in the GP-Out-of-Programme outreach in rural public health facilities in Limpopo. According to this article, the general medical practitioners believed that their clinical skills and competencies were enhanced by the outreach programme. The other article explored the factors that influence the general migration of anatomical pathologists in KwaZulu-Natal. According to this research article, the pathologists felt that not being acknowledged and respected were the greatest factors influencing their decision to migrate. In another article within this theme it was found that pharmacists in Limpopo were willing to be involved in clinical ward views in public hospitals. In another research article it was found that patients believed that mammographers' personality traits like instilling trust, caring, letting patients feel safe and constructive communication contributed to an optimal mammograph experience for them. In the last article within this theme the research results indicated

Peer review under responsibility of Johannesburg University. that nutrition knowledge, food safety and hygiene practices of child and youth care workers could be improved.

\section{Research on health challenges people experience}

Seven of the published articles could be categorised into this theme. Three of the articles dealt with research on aggression and violence. One of these articles examined the effect of trauma on survivors of sexual assault compared to the survivors of intimate violence in Limpopo. Sexual assault was experienced by participants as personal, and intimate violence as interpersonal. The second article dealt with the effect of psychological violence in the workplace on health. It was found that psychological violence impacted on the person as a whole and was unhealthy. The third article examined learners' experiences of teachers' aggression in a secondary school. Learners described being belittled, being emotionally and verbally abused, as well experiencing fear and anger. Two other articles dealing with health challenges focused on the effects of being ill. One article indicated that individuals with multiple sclerosis in the Western Cape felt that they were dependent on others and had lost their freedom. In the last article dealing with health challenges it was found that patients believed that poverty and difficulty in obtaining financial aid and food security were contributing factors with regard to the low tuberculosis cure rates in the greater Giyani health care facilities. The last two articles addressed research on children and aging. The research article on children investigated the prevalence of stunting, wasting and underweight in Grade 1 children and the impact on their health. The last article explored participants' perceptions on menopause and aging in rural villages in Limpopo. It was found that the participants in this study believed that as soon as a woman stopped menstruating she was old. A recommendation was that from adolescence onwards girls should be taught that menopause was an aspect of aging.

\section{Research on treatment of health challenges}

Six published articles fell under this theme. The process to develop and test the Gamma nursing measure was described 
in one article. This instrument could be used by nurses to score patients' abilities to live independently. In another article the therapeutic use of music as experienced by cardiac surgery patients in an intensive care unit was explored. The patients felt that music contributed to their healing. Another article looked at adherence therapy and motivational interviewing techniques during in-hospital stays as interventions to promote psychiatric patients' compliance with mental health regimes. In another article interventions to reduce stigma attached to mental illness were described. The interventions included web-based approaches, printed educational materials, documentary and anti-stigma films as well as live and video performances. The results of research on the effect of rehabilitation on intellectually disabled people were described in another article. The article described how rehabilitation could improve activities of daily living in people with mild and moderate disabilities, including self-care, communication and improvement of cognition. The last article within this theme focused on the implementation practices of the directly-observed treatment strategy (DOTS) in a hospital in the eThekwini health district. It was found that DOTS was not implemented according to World Health Organization standards.

\section{Research on issues in women's health}

Six of the published articles could be categorised under this theme. Four of the six articles focused on research on pregnant women. One of the articles explored midwives' perceptions regarding psychosocial risk assessment during antenatal care. The results indicated that there was a high prevalence of psychosocial problems experienced by pregnant women. In another article community-physician-based care was compared to hospital-based ante-natal care with regard to patient satisfaction. Hospital-based ante-natal care by midwives was experienced by patients as less satisfactory because of the long waiting time before they were seen. The psychological experiences of women who survived HELLP (haemolysis, elevated liver enzymes and low platelet count) were described in another article. The results showed that pregnant women experienced a lack of knowledge about HELLP, and that there was a need to assign blame and shift their focus. The last two articles within this theme focused on challenges to women's health. One article examined the knowledge of Buffalo City female learners about abortion legislation. It was found that female learners had poor knowledge about abortion legislation and that there were misunderstandings about the issue. The other article looked at women's perceived susceptibility to cervical cancer and the utilisation of cervical cancer screening services in Malawi. The results showed that Malawian women lacked awareness about their susceptibility to cervical cancer and required information about available cancer screening services.

\section{Research regarding HIV and AIDS}

Five articles on this theme were published. One article reported on an investigation of patients' knowledge and beliefs regarding antiretroviral treatment (ART) and factors associated with adherence in Mpumalanga. Results indicated that patients living with HIV believed that ART was harmful to their bodies and they sometimes missed dosages of ART for a number of different reasons. Another article addressed the issue of the prevention of mother-to-child transmission of HIV with regard to data completeness and accuracy assessment in health facilities in the Nkangala District. Results showed that there was a need for improvement in data completeness and accuracy assessment. Another article reported on a comparison of HIV patterns in two provinces in South Africa. Results showed a decline in HIV in youth in KwaZulu-Natal compared with an increase in HIV in youth in the Western Cape. Recommendations included practices of sexual abstinence and condom use for youth in the Western Cape. In another article refugees' perceptions were examined regarding HIV and AIDS in Ba-Phalaborwa Municipality in Limpopo District. Refugees demonstrated low levels of knowledge and believed in myths. The last article dealing with this theme explored health workers' perspectives on the implementation of an integrated medical male circumcision strategy in KwaZulu-Natal. The researchers reported that medical male circumcision was highly accepted by health workers. However, health workers also believed that medical male circumcision would discourage condom use and cause stigma associated with non-circumcised HIV-positive males.

Lastly, the research designs of the research reported in these articles were of a qualitative, quantitative, mixed and systematic review type. There was thus a balance in these designs without any one paradigm dominating.

From January 2015 Elsevier will be HSAG's new publisher and we look forward to participating on an international platform. We would also like to take this opportunity to thank AOSIS for their excellent service as our publisher up to 2015.

Marie Poggenpoel, Professor: Nursing Science, Editor-inChief Department of Nursing, University of Johannesburg, Sixth Floor, West Wing North, John Orr Building, 6104e, C/O Beit and Nind Street, Doornfontein, South Africa

*Tel.: +27 115596686. E-mail address: mariep@uj.ac.za

Available online 19 June 2015

http://dx.doi.org/10.1016/j.hsag.2015.04.002 1025-9848/Copyright $\odot$ 2015, The Author. Production and hosting by Elsevier B.V. on behalf of Johannesburg University. This is an open access article under the CC BY-NC-ND license (http:// creativecommons.org/licenses/by-nc-nd/4.0/). 